\title{
Role of Advanced Imaging in Gasserian Neurolysis in Head and Neck Cancer with Skull Base Invasion
}

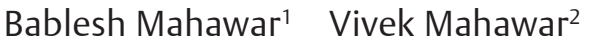 \\ ${ }^{1}$ Department of Pain Management and Supportive Care, Rajiv \\ Gandhi Cancer Institute and Research Center, New Delhi, India \\ 2Department of Radiology and Diagnostic Imaging, Rajiv Gandhi \\ Cancer Institute and Research Center, New Delhi, India
}

\begin{abstract}
Address for correspondence Bablesh Mahawar, MBBS, DNB, FIPM, Department of Pain Management and Supportive Care, Rajiv Gandhi Cancer Institute and Research Center, New Delhi, India (e-mail: drmahawarbablesh@gmail.com).
\end{abstract}

Asian J Oncol 2019;5:86-88

\begin{abstract}
Keywords

- trigeminal neuropathy

- Gasserian neurolysis

- foramen ovale

- glycerol

Introduction A 46-year-old female with a postoperated case of carcinoma left upper alveolus diagnosed in March 2018, with recurrence, was presented to the hospital. She had lancinating pain around left facial pain, which gradually increased over 10 months. On numerical rating scale, her pain was 9 on 10. Her chief complaint was continuous dull pain above and below the left eye, with sudden, brief, paroxysmal episodes of intolerable burning and stabbing type of pain. This episodic pain lasted for 30 to 40 seconds. On examination, she had left III, V, and VI nerve palsy with ptosis. Magnetic resonance imaging of face and brain revealed an enhancing infiltrative mass in left maxillary sinus extending to base of skull. Clinical diagnosis of left-sided painful trigeminal neuropathy in ophthalmic and maxillary (V1 and V2) division was made.

Aim This study was aimed to relieve patient's pain and improve her quality of life.

Materials and Methods Gasserian neurolysis was successfully completed under computed tomography (CT) guidance because of the distortion of skull base anatomy using a $22-\mathrm{G}, 10-\mathrm{cm}$ long spinal needle directed towards foramen ovale (FO) from a lateral approach.

Conclusion CT-guided identification of foramen ovale can be labeled as a novel method of locating FO for precise percutaneous techniques to the trigeminal ganglion in advanced head and neck cancers.
\end{abstract}

\section{Introduction}

Among the acute and chronic pain syndromes, cancer pain is the most significant. The most worried symptom of cancer patients is suffering from pain. Effective control of cancer pain has long been one of the most important and pressing issues about oncology and health care systems because cancer pain dramatically affects patients and their families. Cancer pain is often inadequately managed; as a result, many patients spend the last days of their lives suffering, with great discomfort and disability. Thus, pain management is an essential part of oncologic management. We present a case of a 46 -year-old female with carcinoma on left upper alveolus with painful trigeminal neuropathy (PTN) ${ }^{1}$ and skull base invasion treated successfully by computed tomography (CT) guided Gasserian ganglion neurolysis in local anesthesia (LA).

\section{Case Report}

A 46-year-old female, case of carcinoma on left upper alveolus, was diagnosed in March 2018. Disease recurred in August 2018 proved on histopathology for which she received chemoradiation. Later, she developed severe excruciating left-sided facial pain. She was prescribed opioids at her native place, but she developed opioids induced nausea and vomiting and hospitalized for 6 days. The patient received diagnostic infraorbital and supraorbital nerve block in her native place that did not relieve her symptoms.

The patient presented to the Rajiv Gandhi Cancer Institute for further management and was referred to the pain clinic by the oncology team. She had lancinating pain around the left side of face. The pain gradually increased over 10 months. On numerical rating scale (NRS), her pain was 9 on 10 . Her 


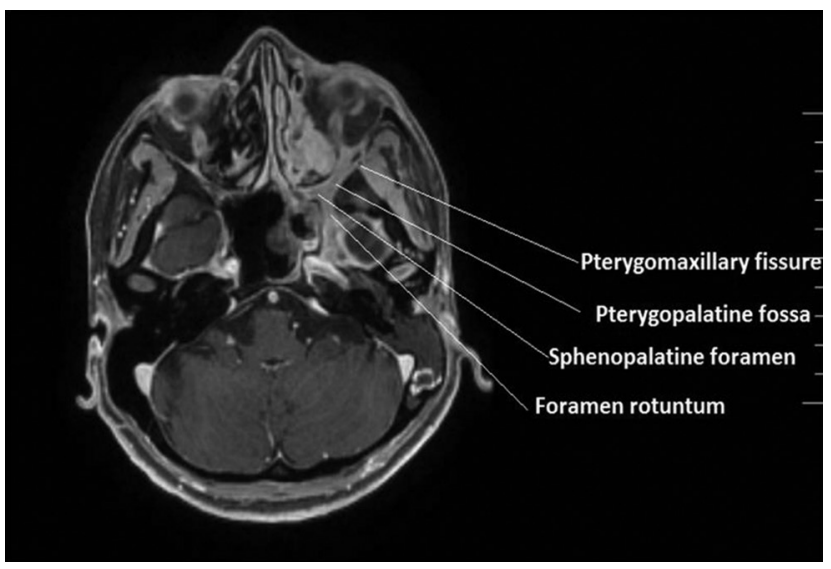

Fig. 1 Magnetic resonance imaging of face and neck showing extent of the tumor.

chief complaints are constant dull pain above and below the left eye, with sudden, brief, paroxysmal episodes of intolerable burning, and stabbing type of pain. This episodic pain lasted for 30 to 40 seconds. On examination, she had left III, $\mathrm{V}$, and VI nerve palsy with ptosis. Magnetic resonance imaging (MRI) of face and brain revealed an enhancing infiltrative mass in left maxillary sinus extending in pterygomaxillary fissure, pterygopalatine fossa, sphenopalatine foramen, foramen rotundum, left cavernous sinus, and Meckel's cave region ( - Fig. 1). Clinical diagnosis of left-sided painful trigeminal neuropathy in ophthalmic and maxillary $\left(V_{1}\right.$ and $\left.V_{2}\right)$ divisions was made. Her concern was to relieve her pain to improve her quality of life.

Neurosurgeon's opinion obtained, the tumor was inoperable due to intracranial and cavernous sinus extension. She was started on opioids but developed profuse vomiting and denied to take opioids further. Giving due consideration on her noncompliance with opioids, she was started on tablet oxcarbazepine $300 \mathrm{mg}$ once a day. As we could not wait for the pharmacological effects of drugs to come, it was decided to take the patient for Gasserian neurolysis under CT guidance because of the distortion of skull base anatomy.

The patient counselled and informed written consent was taken. The coagulation profile was checked and she was called the next day nil per oral. Intravenous access was secured and antibiotic was administered. CT base of skull was done to look for the feasibility of foramen ovale (FO). The patient was placed in a supine position with the head in a neutral position on a CT scanner bed. The needle entry point was marked with a marker and local infiltration with $1 \%$ lidocaine after routine disinfection and draping. A 22-G, $10-\mathrm{cm}$ long spinal needle was directed toward FO from a subzygomatic approach in cranial direction and tip of the needle is placed in the FO with the help of reformatted coronal CT

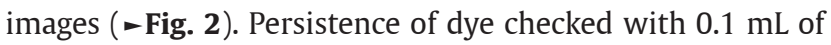

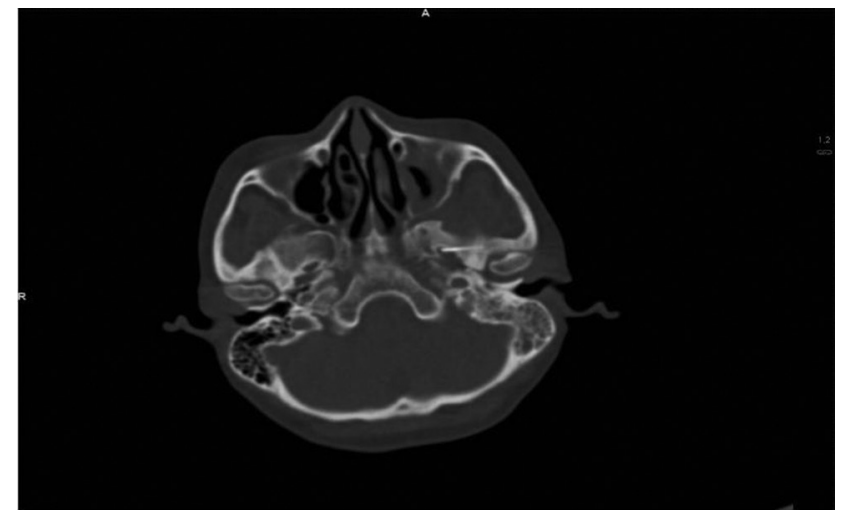

Fig. 2 Computed tomography image showing needle tip inside foramen ovale.

contrast and $0.1 \mathrm{~mL}$ of $0.5 \%$ lignocaine. This doubly ensured us about the location as well as the spread of injectate. It was followed by $0.2 \mathrm{~mL}$ of glycerol and needle was flushed with dexamethasone. The patient observed for 4 hours then discharged from day care and called for follow-up after 5 days. On follow-up, she marked her pain as 1 on 10 and confirmed that she felt a lot better.

\section{Discussion}

Lam et $\mathrm{al}^{2}$ quotes CT-guided pulse radiofrequency ablation invention is an effective and safe technique for medically intractable idiopathic TN patients. Mostly Gasserian neurolysis is routinely done by most of the pain physicians using $\mathrm{X}$-ray or fluoroscopic guidance but, in cases of advanced stages of head and neck cancer with skull base invasion, fluoroscopy has its own limitations in terms of image quality and two-dimensional views. In contrast to this, CT scan provides excellent and direct visualization of FO leading to correct placement of the needle, reducing complications and improving the results. Thus, sometimes in advanced malignancies, CT scores over fluoroscopy.

\section{Conclusion}

1. Pain management in cancer patients is important, ${ }^{3}$ but sometimes a difficult task.

2. When anticancer therapy provides no relief or recurrent pain is refractory to further palliative measures, it is essential to treat the pain symptomatically.

3. The advanced image-guided techniques can increase the precision of the analgesic procedures, improving the results and reducing complications.

4. Thus, CT guided the identification of FO can be labeled as a novel method of locating FO for precise percutaneous techniques to the trigeminal ganglion in advanced head and neck cancers. 


\section{Conflict of Interest}

None declared.

\section{References}

1 Peñarrocha-Diago M, Mora-Escribano E, Bagán JV, Peñarrocha-Diago M. Neoplastic trigeminal neuropathy: presentation of 7 cases. Med Oral Patol Oral Cir Bucal 2006;11(2):E106-E111
2 Lan M, Zipu J, Ying S, Hao R, Fang L. Efficacy and safety of CT-guided percutaneous pulsed radiofrequency treatment of the Gasserian ganglion in patients with medically intractable idiopathic trigeminal neuralgia. J Pain Res 2018;11:2877-2885

3 Aucoin JA, Muscat P, Lu-Emerson C. Painful trigeminal neuropathy as a rare but important complication of head and neck cancer: a case report and review. J Neurol Spine 2017;2(1):1007 